\title{
Treatment of Giant Left Atrial Diverticulum: A Case Report and Literature Review
}

\author{
Hongmu Li, Sunang Yan, Haibo Liu* \\ Department of Cardiovascular Surgery, Quanzhou First Hospital of Fujian Medical University, Quanzhou, China
}

\section{ABSTRACT}

In this paper, we present a giant left atrial diverticulum (LAD) in a 10-year-old girl, whose three-dimensional (3D) image reconstruction was used to help diagnosis and surgical positioning. Previously reported cases were reviewed, and the clinical characteristics of this disease also was summarized to improve the diagnosis and treatment of LAD.

\section{INTRODUCTION}

Left atrial diverticulum (LAD) is a rare malformation. Its exact etiology is unclear, and it frequently is detected on routine chest $\mathrm{x}$-rays without presentation of symptoms. The most useful treatment is surgical resection. This paper reports about a 10-year-old girl diagnosed with giant LAD, whose surgical resection ultimately was aided with threedimensional (3D) image reconstruction.

\section{CASE REPORT}

A 10-year-old girl was presented to our institute with bronchopneumonia. Chest $\mathrm{x}$-ray revealed enlargement of the cardiac silhouette. (Figure A) On echocardiographic evaluation, a large cystic cavity was seen communicating with the left atrial, compressing and displacing the left ventricle toward the right side. (Figure B) Furthermore, computed tomography angiography with $3 \mathrm{D}$ reconstruction imaging confirmed the diagnosis of left atrial diverticulum. (Figure C) To exclude the possibility of malignant tumors, PET-CT also performed before operation suggested the left atrial diverticulum did not increase in metabolism. (Figure D) An isolated left atrial diverticulum resection was performed without extracorporeal circulation. (Figure E) On pathology, the diverticulum was identified as myocardium with mild hyperplasia of interstitial fibrous tissue and a few inflammatory cell infiltrations. (Figure F) The patient was extubated after 4 hours of ventilation and had an uneventful recovery. Left atrial diverticulum is a rare malformation. The exact etiology still is unclear.

Received December 8, 2019; accepted December 14, 2019.

*Correspondence: Haibo Liu, Department of Cardiovascular Surgery, Quanzhou First Hospital of Fujian Medical University, Quanzhou 362000, China; (e-mail:lbb2233.hot@163.com).
Detailed preoperative examinations, particularly echocardiography and 3D image reconstruction, are a great help in the diagnosis and selection of surgical strategies.

\section{DISCUSSION}

LAD is a rare malformation. Since the first report in 1938, there only have been 30 reports in the world [Gao 2011]. The exact etiology still is unclear. Only one case suggested a genetic predisposition, but this has not been confirmed [Jenni 1981]. It currently is believed the etiology of the left atrium diverticulum may be congenital and acquired. They are considered to be congenital if the diverticulum arise from focal areas of developmental weakness of the atrial wall [Shaher 1972], and acquired if it is secondary to atrial wall damage, such as rheumatic heart disease, tuberculosis, syphilitic cardiomyopathy or surgery [Chockalingam 2003; De Ponti 2013; Ding 2015; Halbertsma 2002].

LAD usually presents asymptomatic, as it frequently is detected on routine chest $\mathrm{x}$-ray incidentally [Genc 2014]. For symptomatic patients, often concomitant mitral regurgitation, arrhythmias, thromboembolism, or compression of the surrounding structures has been described in patients with LAD [Hayashi 2013; Higashida 2018; Holda 2017; Nomura 2008; Tan 2014; Terada 2000]. In addition, atrial septal defect and ventricular septal defect also have been described in patients with LAD [Victor 1984]. Foale and Herzog proposed that echocardiography is a safe and effective method for diagnosing left atrial diverticulum ]Foale 1982; Herzog 2009]. In addition, cardiac CT and multidetector CT also helps effectively confirm LAD [Abbara 2009; Patel 2013; Wan 2009].

As LAD usually does not become clinically apparent until complications such as cardiac arrhythmias and sudden death occur, early diagnosis and surgical excision is mandatory. Qiang Chen [Chen 2017] and Agematsu [Agematsu 2009] suggested that early surgical excision should be performed to decrease systemic thrombosis, renew the atrium normal shape, and improve cardiac function, no matter the presence of any symptoms. But Binder et al considered treatments only for symptomatic patients [Binder 2000]. Other management options that have been reported for such large diverticulum are anticoagulation with observation and heart transplantation [Lei 2014].

Although our patient was asymptomatic, surgical resection was performed without extracorporeal circulation because of the potential risk of thromboembolism and arrhythmia. The 

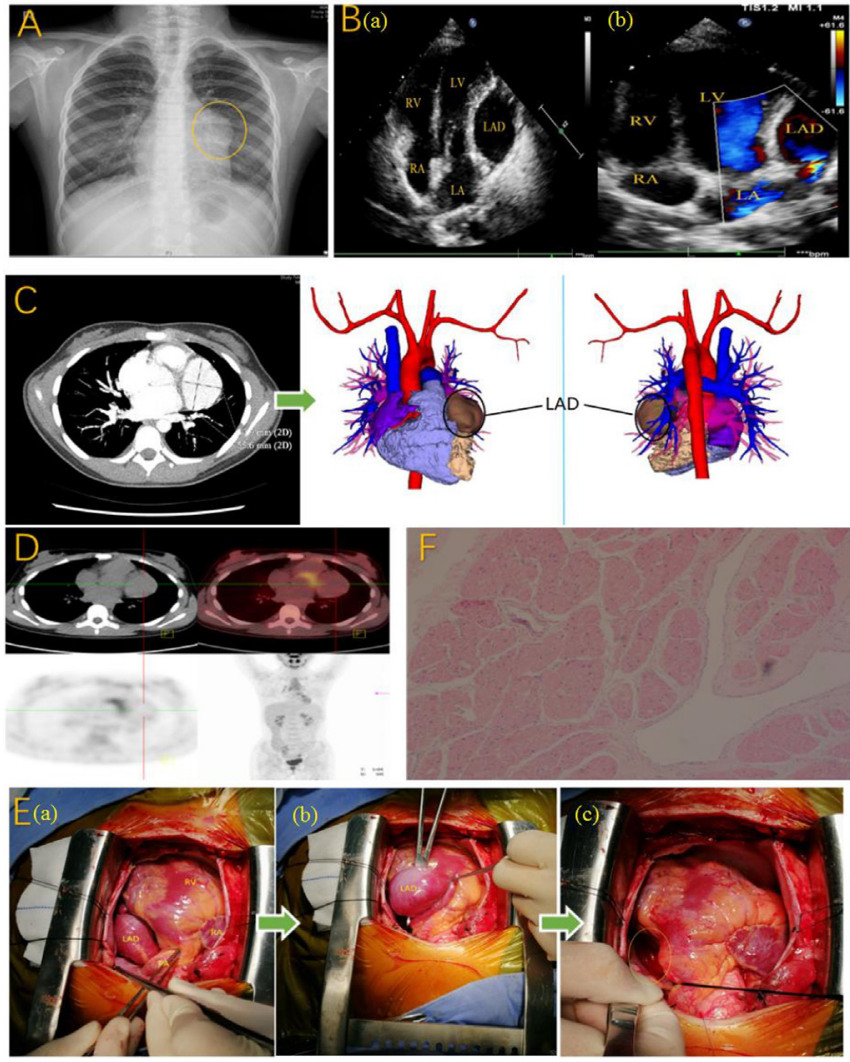

A. Chest x-ray revealed enlargement of the cardiac silhouette. B. Twodimensional echocardiogram (a) and doppler echocardiogram (b) showed the diverticulum. LA: left atrium; RA: right atrium; LV: left ventricle; RV: right ventricle; LAD: left atrial diverticulum. C. The diverticulum was approximately $42.9 \mathrm{~mm} \times 52.3 \mathrm{~mm}$ measured by Computed tomography angiography with three-dimensional reconstruction. Besies, $3 D$ reconstructed image showed $L A D$ position and adjacent structure. LAD: left atrial diverticulum. D. PET-CT suggested left atrial diverticulum no increase in metabolism. E. Operative photographs showing: (a) a large diverticulum from the lateral wall of the left atrium. (b) Ligation of the diverticulum along the neck and resected completely. (c) a cavity left after the diverticulum is removed. LA: left atrium; RA: right atrium; LV: left ventricle; RV: right ventricle; LAD: left atrial diverticulum. F. Histopathological appearance showing myocardium was identified in the wall of the diverticulum with mild hyperplasia of interstitial fibrous tissue and a few inflammatory cell infiltrations.

3D image reconstruction played an important role in diagnosing LAD and surgical positioning. This is the first such case worldwide. The patient had a good postoperative result.

\section{REFERENCES}

Abbara S, Mundo-Sagardia JA, Hoffmann U, Cury RC. 2009. Cardiac CT assessment of left atrial accessory appendages and diverticula. AJR. American journal of roentgenology 193, 807-812.

Agematsu K, Okamura T, Ishihara K, Kurosawa H. 2009. Remarkable giant right atrial diverticulum in asymptomatic patient. Interactive cardiovascular and thoracic surgery 8, 705-707.
Binder TM, Rosenhek R, Frank H, Gwechenberger M, Maurer G, Baumgartner H. 2000. Congenital malformations of the right atrium and the coronary sinus: an analysis based on 103 cases reported in the literature and two additional cases. Chest 117, 1740-1748.

Chen Q, Cao H, Zhang GC, Huang XM. 2017. A Giant Left Atrial Diverticulum Diagnosed by Transesophageal Echocardiogram and Multidetector Computed Tomography: A Case Report and Brief Review of the Literature. The heart surgery forum 20, E082-E084.

Chockalingam A, Alagesan R, Nandakumar M, Gnanavelu G. 2003. Massive left atrial appendage aneurysm presenting as supraventricular tachycardia. Indian heart journal 55, 379-381.

De Ponti R, Lumia D, Marazzi R, Mameli S, Doni LA, De Venuto G. 2013. Left atrial diverticula in patients undergoing atrial fibrillation ablation: morphologic analysis and clinical impact. Journal of cardiovascular electrophysiology 24, 1232-1239.

Ding X, Zhu M, Wei C, Zhu H, Zhang X, Qu X. 2015. Double cardiac diverticula after pericardectomy. International journal of cardiology 201, 449-453.

Foale RA, Gibson TC, Guyer DE, Gillam L, King ME, Weyman AE. 1982. Congenital aneurysms of the left atrium: recognition by cross-sectional echocardiography. Circulation 66, 1065-1069.

Gao C, Wang R, Wang G, Wang Y. 2011. Giant left atrial diverticulum. Journal of cardiac surgery 26, 70 .

Genc B, Solak A, Kantarci M, Bayraktutan U, Ogul H, Yuceler Z. 2014. Anatomical features and clinical importance of left atrial diverticula: MDCT findings. Clin Anat 27, 738-747.

Halbertsma FJ, van Oort A, van der Staak. 2002. Cardiac diverticulum and omphalocele: Cantrell's pentalogy or syndrome. Cardiology in the young 12, 71-74.

Hayashi T, Naito S, Kumagai K, Ohshima S, Hachiya H, Hirao K. 2013. Ventricular tachycardia associated with a giant right atrial diverticulum. Journal of the American College of Cardiology 62, 2341.

Herzog BA, Husmann L, Jenni R, Kaufmann PA. 2009. Diverticulum of the posterior left atrial wall. Echocardiography 26, 471-472.

Higashida A, Hoashi T, Sakaguchi H, Ichikawa H. 2018. Neonatal repair of left atrial diverticulum with gigantic thrombus without cardiopulmonary bypass. General thoracic and cardiovascular surgery 66, 232-234.

Holda MK, Koziej M, Wszolek K, Pawlik W, Krawczyk-Ozog A, Sorysz D. 2017. Left atrial accessory appendages, diverticula, and left-sided septal pouch in multi-slice computed tomography. Association with atrial fibrillation and cerebrovascular accidents. International journal of cardiology 244, 163-168.

Jenni R, Goebel N, Schneider L, Krayenbuhl HP. 1981. [Idiopathic familial right atrial dilatation]. Schweizerische medizinische Wochenschrift 111, 1565-1572.

Lei Q, Guo HM, Luo ZC. 2014. Surgical treatment of giant left atrial diverticulum in an adult. The Annals of thoracic surgery 98, 1820-1821.

Nomura K, Matsumura Y, Shinohara G, Nakamura Y. 2008. A 4-year-old girl with giant left atrial diverticulum resulting in severe mitral regurgitation. Cardiovascular pathology : the official journal of the Society for Cardiovascular Pathology 17, 254-255.

Patel SN, French A, Mathias H, Lyen S, Hamilton MC, Manghat NE. 2013. Presence of left atrial diverticula, accessory appendages, and normal variant pulmonary venous anatomy diagnosed using MDCT and adverse outcomes following radiofrequency catheter ablation therapy in patients with drug-refractory atrial fibrillation: an exploratory study. Clinical radiology 68, 762-769. 
Shaher RM, Alley R, Anis W, Mintzer J. 1972. Congenital enlargement of the left atrium. The Journal of thoracic and cardiovascular surgery 63, 292-299.

Tan C, Han W, Liu X, Hu X, Liu J, Cui J. 2014. Electrophysiological characteristics of left atrial diverticulum in patients with atrial fibrillation: electrograms, impedance and clinical implications. International journal of cardiology 176, 48-54.

Terada H, Tanaka Y, Kashima K, Sannou K, Arima T. 2000. Left atrial diverticulum associated with severe mitral regurgitation. Japanese circulation journal 64, 474-476.

Victor S, Ravindran P, Maragatham S, Chockalingam V, Lakshmikanthan C. 1984. Congenital diverticulum of muscular interventricular septum in association with atrial septal defect. Indian heart journal 36, 401-402.

Wan Y, He Z, Zhang L, Li B, Sun D, Fu F. 2009. The anatomical study of left atrium diverticulum by multi-detector row CT. Surgical and radiologic anatomy : SRA 31, 191-198. 